\title{
Über die Phosphorylierung von Diäthanolamin durch Alkalische Phosphatase
}

\author{
Von R. Zech, MARIA Grote ${ }^{1}$ ), K. ZürCher und R. Schlaeger \\ Aus dem Strablenbiochemischen Laboratorium im Pbysiologiscb-chemischen Institut und der Abteilung für Kliniscbe Chemie \\ der Medizinischen Klinike der Universität Göttingen
}

(Eingegangen am 23. Juli/August 1973)

Es wird die Umsetzung von 4-Nitrophenylphosphat durch cholestatisches Ratten- und Humanserum in Diäthanolaminpuffer unterschiedlicher Konzentration bei verschiedenen pH-Werten untersucht. Es konnte eine Phosphorylierung des Diäthanolamin durch 4-Nitrophenylphosphat und Alkalische Phosphatase nachgewiesen werden.

\section{The phosphorylation of diethanolamine by alkaline phosphatase}

The action of cholestatic serum of man and rat on 4-nitrophenylphosphate and diethanolamine was studied at different $\mathrm{pH}$ values in different concentrations of diethanolamine buffer. A phosphorylation of diethanolamine by 4-nitrophenylphosphate und alkaline phosphatase was shown.

Die Aktivität der Alkalischen Phosphatase (Orthophosphorsäuremonoester-phosphohydrolase EC 3.1.3.1) wird im optimierten kinetischen Test nach RICK et al. (1) mit $10 \mathrm{mmol} / 1$ Nitrophenylphosphat in $1 \mathrm{~mol} / 1$ Diäthanolamin-Puffer $\mathrm{pH} 9,8$ und $0,5 \mathrm{mmol} / \mathrm{l}$ Magnesiumchlorid gemessen. Als $\mathrm{MaB}$ für die hydrolytische Aktivität wird die Extinktion des freigesetzten 4Nitrophenolat-Anions bei 400 oder $405 \mathrm{~nm}$ bestimmt. Gegenüber anderen Methoden mit 2-Glycerophosphat oder anderen Phosphatestern in verschiedenen Puffersystemen mit kolorimetrischer Bestimmung des freigesetzten anorganischen Phosphats sowie den Methoden mit 4-Nitrophenylphosphat in Carbonat-, Glycin- oder Piperazinpuffer ergibt der Nachweis in 1 oder $2 \mathrm{~mol} / \mathrm{l}$ Diäthanolamin eine zwei- bis sechsfach höhere Phosphataseaktivität. Der Einfluß des Diäthanolamin auf die Kinetik der 4-Nitrophenylphosphat-Umsetzungen durch Alkalische Phosphatase wird im folgenden beschrieben.

\section{Material und Methoden}

Rattenserum: $220 \mathrm{~g}$ schwere weibliche Wistar-Ratten wurden nach 24-stündiger Choledochusligatur in leichter Äthernarkose durch Herzpunktion entblutet. Das Serum wurde gewonnen und gepoolt bei $-25^{\circ} \mathrm{C}$ aufbewahrt.

Ikterisches Humanserum stammte von Patienten mit extrahepatischer Cholestase, die Alkalische Phosphatase betrug 600-800 U/1 im optimierten Test. Die Transaminasen waren im Normbereich. Vor Gebrauch wurden die Seren $2 \times 90 \mathrm{~min}$ gegen $1 \mathrm{mmol} / 1$ $\mathrm{MgCl}_{2}$ und $0,1 \mathrm{~mol} / \mathrm{l} \mathrm{NaCl}$ bei pH 7 zür Herabsetzung des Phosphatblindwertes dialysiert.

Diäthanolamin zur Synthese, 4-Nitrophenylphosphat, Magnesiumchlorid p. a., Zinkchlorid p. a., Zinnchlorid p. a., Butanol-(1) p. a. und Ninhydrin p. a. von der Fa. Merck, Dünnschichtfertigfolien Polygram Sil G $10 \times 20 \mathrm{~cm}$ der Fa. Macherey und Nagel, DEAF Sephadex A 25 von der $F_{a}$. Pharmacia, Ammoniummolybdat von Riedel de Haen und $3 \mathrm{~mol} / 1$ Trichloressigsäure von der $\mathrm{Fa}$. Boehringer.

Die Bestimmung von anorganischem Phosphat erfolgte in einem V.olumen von $0,5 \mathrm{ml}$, eine Enteiweißung erfolgte durch Zusatz von $0,2 \mathrm{ml} 3 \mathrm{~mol} / 1$ Trichloressigsäure. Nach Zentrifugation wurden $0,5 \mathrm{ml}$ Uberstand mit $0,2 \mathrm{ml}$ einer frisch hergestellten Lösung aus gleichen Teilen $5 \mathrm{~mol} / 1$ Schwefelsäure und Ammoniummolybdatlösung $(54 \mathrm{~g} / \mathrm{l})$ versetzt. Die Reduktion des Farbkomplexes er- folgte durch Zusatz von $0,2 \mathrm{ml}$ einer sauren Zinn-(II)-ChloridLösung, die täglich frisch aus einer Stammlösung ( $400 \mathrm{~g} / \mathrm{l} \mathrm{kon-}$ zentrierter Salzsäure) durch 400-fache Verdünnung in $\mathrm{H}_{2} \mathrm{O}$ hergestellt wurde. Eine Reihe der später getesteten Inkubationslösungen beeinflußt die Phosphatbestimmung, es wurde deshalb jeweils für die verschiedenen Inkubationsbedingungen täglich eine Eichung vorgenommen.

Die Bestimmung von organiscbem Phosphat erfolgte nach der Methode von BartLetr (2).

Der Nachweis der Alkalischen Phosphatase erfolgte im optimierten kinetischen Test (1) oder in Inkubationsansätzen von $5 \mathrm{ml}$ in Gegenwart von $1 \mathrm{mmol} / 1 \mathrm{MgCl}_{2}$ und $0,1 \mathrm{mmol} / 1 \mathrm{ZnCl}_{2}$; $\mathrm{zu}$ bestimmten Zeiten wurden $0,5 \mathrm{ml}$. Proben entnommen und die Extinktion bei $400 \mathrm{~nm}$ als $\mathrm{Maß}$ für die Menge des freigesetzten 4-Nitrophenol bestimmt sowie anschließend die Konzentration des anorganischen Phosphats gemessen.

Der $p H$-Wert der Inkubationsansätze wurde zu Anfang und zu Ende des Versuchs mit dem pH-Meter 25 der Fa. Radiometer Kopenhagen kontrolliert.

Die Darstellung des phosphorylierten Diätbanolamins exfolgte aus einem Inkubationsansatz mit dem doppelten Volumen $(10 \mathrm{ml})$ in $1 \mathrm{~mol} / 1$ Diäthanolamin und $50 \mathrm{mmol} / \mathrm{l}$ 4-Nitrophenylphosphat in Gegenwart von $1 \mathrm{mmol} / 1$ Magnesiumchlorid bei $\mathrm{pH}$ 9. Mit $400 \mu$ l ikterischem Rattenserum wurde der Ansatz $17 \mathrm{~h}$ inkubiert, nach dieser Zeit war 15\% des 4-Nitrophenylphosphats umgesetzt, wobei nur $5 \%$ als anorganisches Phosphat erschien. Der gesamte Inkubationsansatz wurde dann über eine $2,5 \times 56 \mathrm{~cm}$ große Säule mit dem Anionenaustauscher DEAE Sephadex A 25 in der OH-Form gegeben und mit $\mathrm{H}_{2} \mathrm{O}$ nachgewaschen. Die Elution erfolgte in $10 \mathrm{mmol} / \mathrm{l}$ Acetat-Puffer $\mathrm{pH} \mathrm{4}$. Das Eluat wurde eingefroren und in der Gefriertrocknung getrocknet. Wir erhielten ein gelbliches, kristallines Pulver.

Die dïnnschicbtcbromatographische Trennung erfolgte auf Polygram Sil G $10 \times 20 \mathrm{~cm}$ in einem Gemisch aus 50 Teilen Butanol-(1), 20 Teilen Eisessig und 50 Teilen Wasser. Nach einer Laufzeit von $6 \mathrm{~h}$ wurden die Chromatogramme mit Ninhydrinspray gefärbt, und in den herausgekratzten Flecken das anorganische und organische Phosphat bestimmt.

\section{Ergebnisse}

Der Einfluß steigender Diäthanolaminkonzentrationen auf die Freisetzung von 4-Nitrophenol aus 4-Nitrophenylphosphat durch ikterisches Rattenserum ist in

1) Die vorliegenden Ergebnisse sind ein wesentlicher Bestandteil der Dissertation von Maria Grote. 


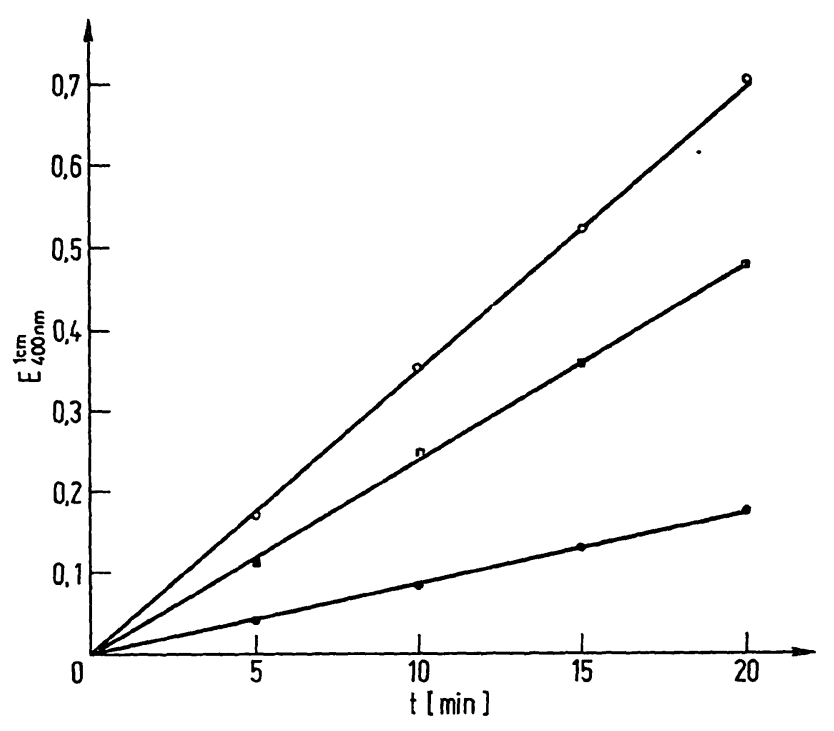

Abb. 1

4-Nitrophenol-Freisetzung aus $1 \mathrm{mmol} / \mathrm{l}$ 4-Nitrophenylphosphat durch Alkalische Phosphatase bei $\mathrm{pH} \mathrm{9,0} \mathrm{in} \mathrm{drei} \mathrm{verschiedenen} \mathrm{Diäthanol-}$ aminkonzentrationen. - - $50 \mathrm{mmol} / \mathrm{l}, \longrightarrow 0,5 \mathrm{~mol} / \mathrm{l}$ und - - - 2 mol/l Diäthanolamin

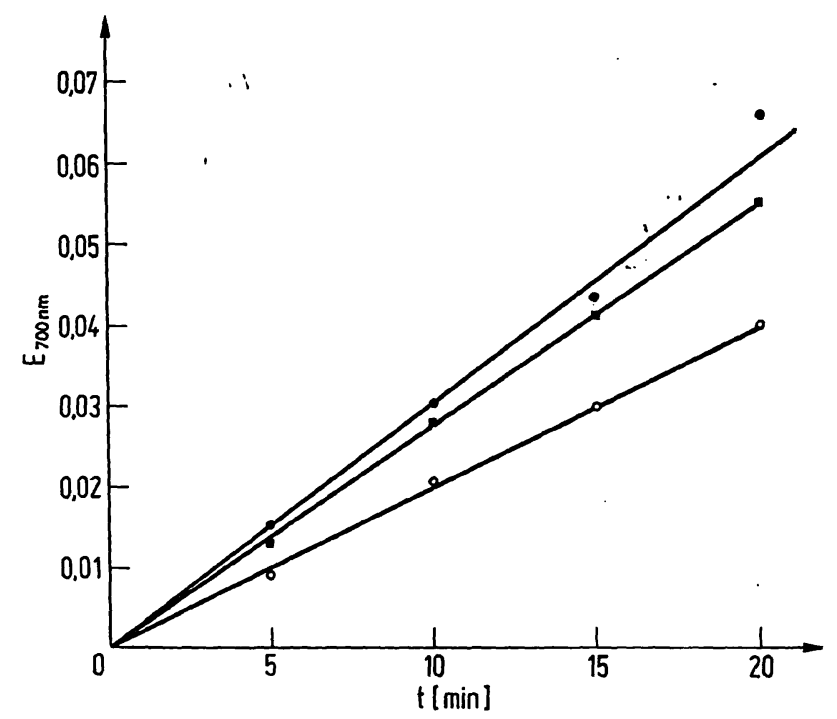

Abb. 2

Phosphatfreisetzung aus $1 \mathrm{mmol}$ 4-Nitrophenylphosphat durch Alkalische Phosphatase bei $\mathrm{pH} \mathrm{9,0}$ in drei verschiedenen Diäthanolaminkonzentrationen. $-\cdot 50 \mathrm{mmol} / \mathrm{l}$, $-0,5 \mathrm{~mol} / 1$ und ○- 2 mol/l Diäthanolamin

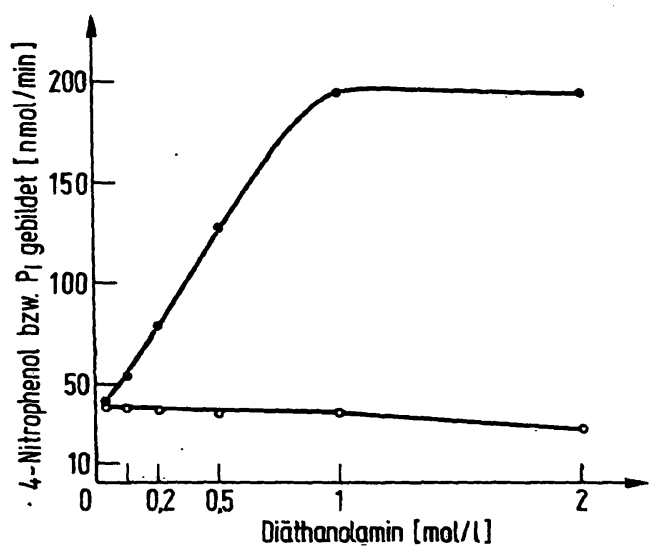

Abb. 3

Einfluß steigender Diäthanolaminkonzentration auf die Umsetzung von 1 mmol/1 4-Nitrophenylphosphat durch $1 \mathrm{ml}$ Serum bei $\mathrm{pH} 9,0$.

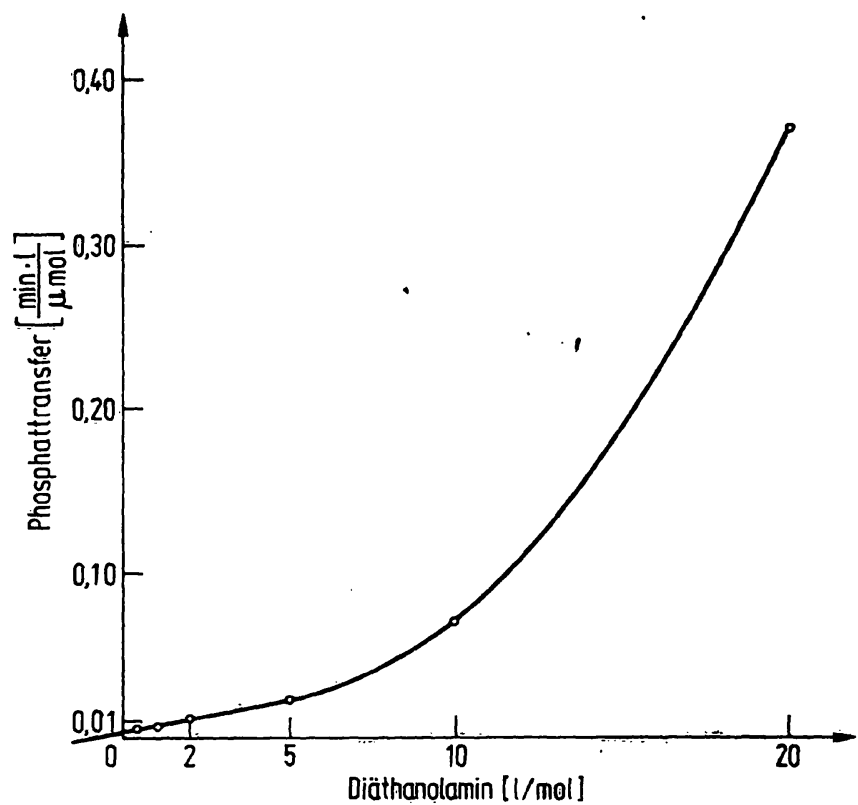

Abb. 4 Reziproke Auftragung des Phosphattransfers von 1 mmol/1 4-Nitro-
phenylphosphat auf Diäthanolamin durch Alkalische Phosphatase bei $\mathrm{pH} 9,0$

Abbildung 1 dargestellt. Durch Diäthanolamin kommt es zu der bekannten starken Aktivierung der Alkalischen Phosphatase, das Aktivitätsverhältnis zwischen $50 \mathrm{mmol} / \mathrm{l}$ Diäthanolamin und $2 \mathrm{~mol} / \mathrm{l}$ Diäthanolamin beträgt 1:4. Wird unter völlig identischen Bedingungen als Maß der Enzymaktivität das freigesetzte anorganische Phosphat gemessen, so ergibt sich ein entgegengesetztes Verhalten: es kommt durch Diäthanolamin zu einer Hemmung der Phosphatfreisetzung (Abb. 2). In Abbildung 3 sind die Freisetzung von 4-Nitrophenol und anorganischem Phosphat in Abhängigkeit von der Diäthanolaminkonzentration dargestellt. Nur in $0,05 \mathrm{~mol} / \mathrm{l}$ Diäthanolamin sind 4-Nitrophenol- und Phosphatfreisetzung äquivalent. Eine Erhöhung der Pufferkonzentration führt zu einer zunehmenden Differenz zwischen beiden Parametern. Eine doppelt reziproke Auftragung dieser Differenz, als Phosphattransfer bezeichnet, gegen die Molarität des Diäthanolamin ergibt im Anfangsteil eine Gerade, bei den niedrigen Pufferkonzentrationen dagegen eine ansteigende Kurve. Die pH-Abhängigkeiten der 4Nitrophenol- und Phosphatfreisetzung in $1 \mathrm{~mol} / \mathrm{l}$ Diäthanolamin verhalten sich unterschiedlich, sie sind in Abbildung 5 aufgetragen. Die Phosphatfreisetzung zeigt ein relativ schmales Optimum im Bereich von $\mathrm{pH} 10$, die 4Nitrophenolabspaltung ergibt ein sehr breites Maximum zwischen $\mathrm{pH} 9$ und $\mathrm{pH}$ 10. Die Differenz zwischen beiden Kurven, der Phosphattransfer, ergibt ein $\mathrm{pH}-$ Optimum bei $\mathrm{pH} 9,0$.

Das unterschiedliche Verhalten der Phosphatfreisetzung und der Abspaltung des 4-Nitrophenol in A $\mathrm{b}$ hängigkeit von der Diäthanolaminkonzentration interpretieren wir als Phosphattransfer auf Diäthanolamin. Dieser Transfer konnte, wie Tabelle 1 zeigt, auch im optimierten Test mit ikterischem Humanserum gezeigt werden. 


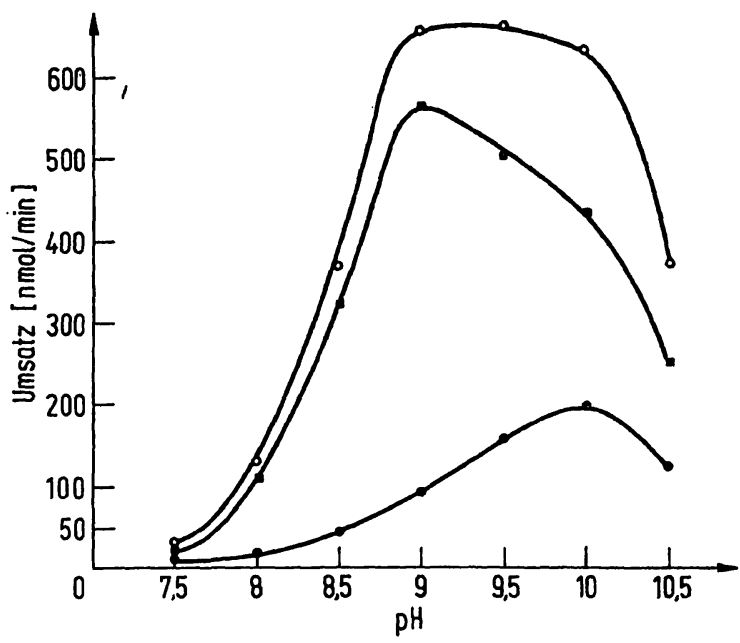

Abb. 5

pH-Abhängigkeit der Umsetzung von 1 mmol/1 4-Nitrophenylphosphat durch Alkalische Phosphatase in $1 \mathrm{~mol} / 1$ Diäthanolamin. Freisetzung von anorganischem Phosphat, o- - - 0 Freisetzung von 4-Nitrophenol, $1-\square-\square$ Phosphattransfer

Tab. 1

Einfluß von Diäthanolamin auf die Umsetzung von 4-Nitrophenylphosphat durch ikterisches Humanserum im optimierten Test pH 9,8, 4-Nitrophenylphosphat $10 \mathrm{mmol} / 1, \mathrm{MgCl}_{2}, 0,5 \mathrm{mmol} / 1$

\begin{tabular}{cccc}
\hline $\begin{array}{c}\text { Diäthanolamin } \\
{[\mathrm{mol} / \mathrm{l}]}\end{array}$ & 4-Nitrophenol & $\begin{array}{c}\mathrm{P}_{1} \\
{[\mu \mathrm{mol} / \mathrm{l} \cdot \mathrm{min}]}\end{array}$ & Phosphattransfer \\
\hline 0,1 & 480 & 461 & 19 \\
1,0 & 1170 & 893 & 277 \\
\hline
\end{tabular}

Das durch Alkalische Phosphatase phosphorylierte Diäthanolamin konnte durch Ionenaustauschchromatographie isoliert werden.

Im Dünnschichtchromatogramm können das phosphorylierte Diäthanolamin und freies Diäthanolamin ge-

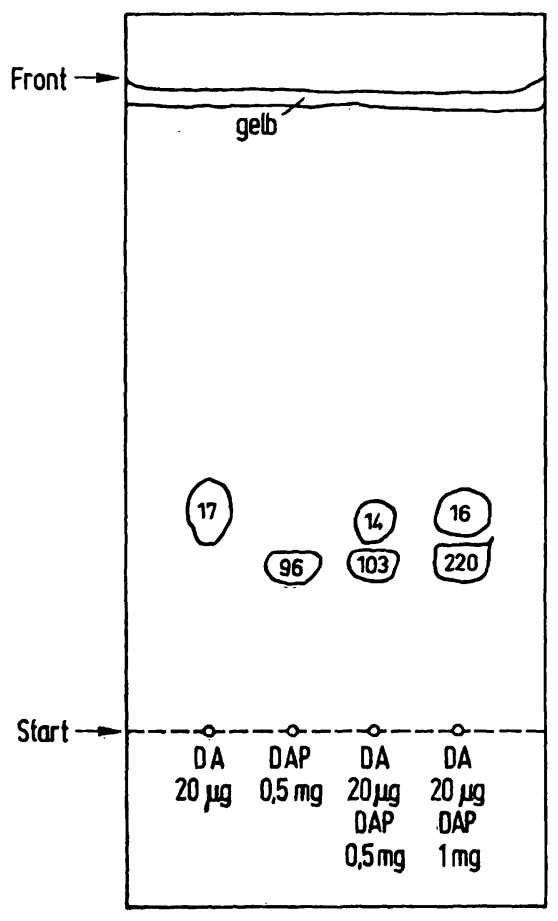

Abb. 6

Dünnschichtchromatographie von Diäthanolamin (DA) und Diàthanolaminphosphat (DAP). Anfärbung mit Ninhydrin. Die Zahlen präparierten Proben an. Unbeschickte Platten ergaben 13 nMol

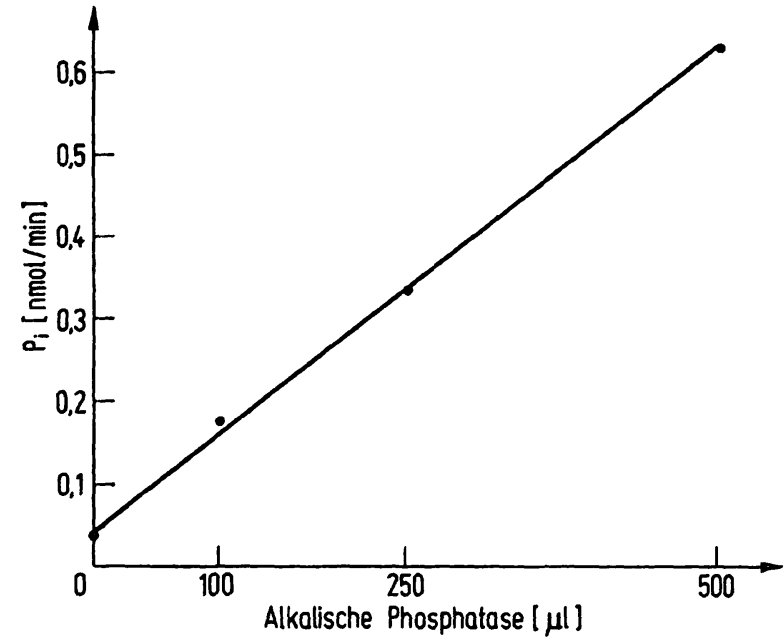

Abb. 7 Hydrolyse von Diäthanolaminphosphat durch Alkalische Phospha-
tase in $50 \mathrm{mmol} / \mathrm{I}$ Diäthanolamin pH 9,0 . Testvolumen $5,0 \mathrm{ml} \mathrm{mit}$ $0,5 \mathrm{mg}$ Diäthanolaminphosphat

trennt und mit Ninhydrin angefärbt werden, in Abbildung 6 ist ein solches Chromatogramm dargestellt. Die Zahlen in den Flecken geben die nMol Phosphat nach Veraschung der extrahierten Proben an. Das durch die Alkalische Phosphatase durch Phosphattransfer entstandene phosphorylierte Diäthanolamin ist seinerseits ein gutes Substrat für die hydrolytische Aktivität der Alkalischen Phosphatase. Abbildung 7 zeigt die Freisetzung von anorganischem Phosphat aus phosphoryliertem Diäthanolamin in Abhängigkeit von der Enzymmenge.

\section{Diskussion}

Der durch Phosphohydrolasen katalysierte Phosphattransfer von einem geeigneten Phosphatdonator auf einen Acceptor wurde erstmals vor etwa 25 Jahren beschrieben (3). Dieser sogenannte "direkte Transfer" würde nach heutiger Enzymsystematik einer echten Transferaseaktivität entsprechen, während der 1928 von $\mathrm{KAY}_{\mathrm{A}}$ (4) beschriebene sogenannte ,indirekte Transfer" aus sehr hohen Konzentrationen von anorganischem Phosphat und Acceptor zu Phosphatester lediglich eine Umkehr der Hydrolasereaktion der Alkalischen Phosphatase ist.

Über die physiologische Funktion des in der vorliegenden Untersuchung verwendeten Enzyms aus der cholestatischen Leber ist bislang nichts Sicheres bekannt, es hydrolysiert ein breites Spektrum von Phosphatestern (5). Die sogenannten substratspezifischen Phosphohydrolasen (EC 3.1.3.3-3.1.3.19) haben bestimmte metabolische Funktionen, wie die Glucose-6phosphat-phosphohydrolase der Leber in der Gluconeogenese. Sie zeigen im Gegensatz zu den unspezifischen Phosphohydrolasen keine Phosphotransferaseaktivität.Die spezifische Glucose-6-phosphat-phosphatase des Dünndarms macht eine Ausnahme: sie zeigt neben der Hydrolaseaktivität echte Transferaseaktivität (6). Andererseits ist die Funktion dieser Glucose-6-phosphatase im Dünndarm nicht klar, denn der Dünndarm 
macht keine Gluconeogenese, was aus dem Fehlen der Phosphoenolpyrurat-Carboxykinase hervorgeht (7). Möglicherweise spielt die Dünndarm-Glucose-6-phosphatase mit ihrer Hydrolase- und Transferaseaktivität eine Rolle beim aktiven Transport während der Glucoseresorption aus dem Dünndarm.

Die Membranlokalisation der Alkalischen Phosphatase mag ebenfalls in dieselbe Richtung deuten, auf eine Rolle im aktiven Transport bestimmter Metabolite. Dieser Anschauung scheinen die benötigten „un- physiologisch" hohen Acceptorkonzentrationen für den Nachweis der Transphosphorylierung zu widersprechen. Der hier vorliegende Befund, daß auch das Produkt der Transferaseaktivität der Alkalischen Phosphatase, das phosphorylierte Diäthanolamin, ein Substrat für die Hydrolaseaktivität dieses Enzyms ist, zeigt, daß Transfer nur. unter Bedingungen nachgewiesen werden kann, bei denen die, Hydrolaseaktivität gegenüber dem Produkt der Transferaseaktivität gehemmt ist.

(Gl. 1) Diäthanolamin + 4-Nitrophenylphosphat $\underset{\uparrow}{\rightleftharpoons}$ Diäthanolaminphosphat + 4-Nitrophenol Alkalische Phosphatase

$$
\text { Diäthanolaminphosphat }+\mathrm{H}_{2} \mathrm{O} \rightleftharpoons \frac{1}{\rightleftharpoons} \text { Diäthanolamin }+\mathrm{P}_{1}
$$

Das in der ersten Reaktion durch die Transferaseaktivität entstehende phosphorylierte Diäthanolamin kann in der zweiten Reaktion durch die Hydrolaseaktivität der Alkalischen Phosphatase gespalten werden, in der Bilanz zeigt sich dann nur eine Hydrolyse des 4-Nitrophenylphosphats. Durch hohe DiäthanolaminKonzentrationen wird aber Reaktion 1 nach rechts verschoben, während Reaktion 2 nach links verschoben wird, wodurch die Transferaseaktivität, also Gleichung 1 , erst nachgewiesen werden kann. Damit ist der in Abbildung 4 sich ergebende Verlauf des LineweaverBurk-Diagramms erklärt, ein wirklicher $\mathrm{K}_{\mathbf{m}}$-Wert ist aus experimentellen Gründen für die Transferaseaktivität nicht zu ermitteln.

AMADor (8) und MCCOMB und Bower (9) konnten ebenfalls Phosphattransfer durch Alkalische Phosphatase auf verschiedene Puffersysteme nachweisen, es fehlen jedoch kinetische Angaben und der direkte Nachweis einer Transphosphorylierung. Wrrson et al. (10) konnten für Alkalische Phosphatase aus E. coli Transferaseaktivität zeigen, das Produkt des Transfers auf Äthanolamin (Phosphoryläthanolamin) konnte im Aminosäureanalysator nachgewiesen werden.

\section{Literatur}

1. Hausamen, T. U., Helger, R., Rick, W. \& Gross, W. (1967), Clin. Chim. Acta 15, 241-245. - 2. Bartlett, G. R. (1959), J. Biol. Chem. 234, 466-468. - 3. Axelrod, B. (1948), J. Biol. Chem. 176, 295-298. - 4. KAY, H. D. (1928), Biochem. J. 22, 855-866. - 5. KapLAN, M. M. (1972), Gastroenterology 62, 452-467. - 6. LYGRE, O. G. \& NordLIE, R. C. (1968), Bio- chemistry 7, 3214-3226. - 7. UTrer, M. F. (1959), Ann. N. Y. Acad. Sci. 72, 451-461. - 8. Amador, E. (1972), Clin. Chem. 18, 94. - 9. МсСомв, R. B. \& Bowers, G. N. (1972), Clin. Chem. 18, 97-104. - 10. Wilson, J. B., Dayan, J. \& Lyo, K. (1964), J. Biol. Chem. 12, 4182-4185.

P. D. Dr. R. Zech

Physiolog. chem. Institut

Strahlenbiochemisches Laboratorium der Universität Göttingen

Humboldtallee 7 